\title{
Low Frequency plasma wave Analyzer (LFA) onboard the PLANET-B spacecraft
}

\author{
H. Matsumoto ${ }^{1}$, T. Okada ${ }^{2}$, K. Hashimoto ${ }^{1}$, I. Nagano ${ }^{3}$, S. Yagitani ${ }^{3}$, M. Tsutsui ${ }^{4}$, Y. Kasaba ${ }^{2}$, \\ K. Tsuruda ${ }^{5}$, H. Hayakawa ${ }^{5}$, A. Matsuoka ${ }^{5}$, S. Watanabe ${ }^{6}$, H. Ueda ${ }^{7}$, I. Kimura ${ }^{8}$, Y. Kasahara ${ }^{9}$,

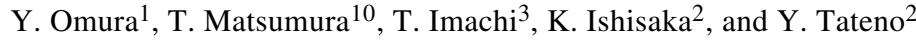 \\ ${ }^{1}$ Radio Atmospheric Science Center, Kyoto University, Uji, Kyoto 611, Japan \\ ${ }^{2}$ Department of Electronics and Informatics, Toyama Prefectural University, Toyama 939-03, Japan \\ ${ }^{3}$ Department of Electrical and Computer Engineering, Kanazawa University, Kanazawa 920, Japan \\ ${ }^{4}$ Institute for Computer Sciences, Kyoto Sangyo University, Kyoto 603, Japan \\ ${ }^{5}$ Institute of Space and Astronautical Science, Sagamihara 229, Japan \\ ${ }^{6}$ Communications Research Laboratory, Koganei 184, Japan \\ ${ }^{7}$ Department of Electrical and Electronics Engineering, Chiba University, Chiba 263, Japan \\ ${ }^{8}$ Faculty of Information Science, Osaka Institute of Technology, Hirakata 573-01, Japan \\ ${ }^{9}$ Department of Electrical Engineering II, Kyoto University, Kyoto 606, Japan \\ ${ }^{10}$ Toshiba Information System Co. Ltd., Kawasaki 210, Japan
}

(Received August 13, 1997; Revised November 25, 1997; Accepted December 2, 1997)

The Low Frequency plasma wave Analyzer, LFA, on board the PLANET-B spacecraft has been developed to measure the Martian plasma waves. Two orthogonal electric dipole wire antennas, $50 \mathrm{~m}$ tip-to-tip, in the spacecraft spin plane are used to measure plasma waves, dc electric fields, and the spacecraft potential relative to the ambient plasma. The LFA has capability to measure the wave spectrum in the band from $10 \mathrm{~Hz}$ to $32 \mathrm{kHz}$, and to capture the signal waveform in the band from dc to $32 \mathrm{kHz}$ by using a 4 MByte memory. The LFA scientific objectives are to explore the following: (1) Macroscopic plasma environment and boundaries from the solar wind to the ionosphere, (2) Microscopic plasma phenomena induced by the interaction between the solar wind and the Martian atmosphere and the moon Phobos, (3) Generation and propagation of electromagnetic waves, (4) Plasma densities and waves in the nightside ionosphere and tail, and (5) Comparison of Martian plasma waves with those of other planets such as non-magnetized Venus and magnetized Earth.

\section{Introduction}

Measurements of the Martian exosphere have been carried out by spacecraft such as Mariner 4, Mars 2, Mars 3, Mars 5, Viking 1 and Phobos 2. They determined the structure of the bow shock, planetosheath, planetosphere, and characteristic boundaries above the ionosphere (Lundin et al., 1990; Grard et al., 1991; Johnson et al., 1991; Slavin et al., 1991). The Martian plasma environment is characterized by the presence of heavy (oxygen) ions of planetary origin. The mass-loading which occurs in the interaction with the solar wind contributes to the structure and position of the bow shock and other boundaries above the ionosphere (Moses et al., 1991). However, detailed features of these boundaries and of the plasma environment are not well understood. The measurement of plasma waves is important for understanding the solar wind interaction with Martian exosphere because the some processes which couple momentum and energy from the solar wind to the Martian plasma may be due to the wave-particle interactions.

A plasma wave instrument known as PWA has been developed to measure Martian plasma waves on board the PLANET-B spacecraft. The PWA consists of a low frequency plasma wave analyzer (LFA) and a plasma wave and sounder

Copy right (C) The Society of Geomagnetism and Earth, Planetary and Space Sciences (SGEPSS); The Seismological Society of Japan; The Volcanological Society of Japan; The Geodetic Society of Japan; The Japanese Society for Planetary Sciences. experiment (PWS). The LFA measures waves from dc to 32 $\mathrm{kHz}$, which we refer to as the LFA band in this paper, while the PWS measures from $20 \mathrm{kHz}$ to $7 \mathrm{MHz}$ utilizing a sweepfrequency analyzer-type receiver. In the following sections, the scientific objectives and instrument characteristics of the LFA are described.

\section{Scientific Objectives}

The scientific objectives of LFA address the following phenomena:

The macroscopic plasma environment and boundaries from the solar wind to the ionosphere: (a) Plasma parameters in the space surrounding Mars by measuring the plasma waves and spacecraft potential; (b) The structure of the bow shock and the planetopause, and how they are effected by the solar wind ram pressure and solar activity; (c) Remote sensing of the global plasma density variations by electromagnetic wave detection such as the $2 f_{p}$ emission and the nonthermal continuum radiation; and (d) Amount of the cold plasma outflow from the ionosphere. The characteristic frequencies of most of the Martian plasma waves are in the band measured by the LFA. Over the most of the elliptical orbit of the PLANET-B spacecraft, the plasma frequency, $f_{p}(\mathrm{kHz})=1 /(2 \pi) \cdot \sqrt{N e^{2} /\left(\varepsilon_{0} m\right)}=9 \sqrt{N}\left(\mathrm{~cm}^{-3}\right)$ is estimated to be lower than $32 \mathrm{kHz}$ except for the high density regions in the ionosphere (Johnson et al., 1991; Pedersen et al., 1991; Woo and Kilore, 1991). The electron 
gyro-frequency, $f_{c}(\mathrm{~Hz})=1 /(2 \pi) \cdot(|e| B / m)=28 B(\mathrm{nT})$ is also estimated to be lower than $32 \mathrm{kHz}$ (Dubinin et al., 1991), even considering the recent observations of the magnetic field by Mars Global Surveyor (Showstack, 1997).

Microscopic plasma phenomena associated with the interaction between the solar wind and the Martian atmosphere: (a) Contributions of various wave-particle interactions to the mass-loading process at the ionopause; (b) Nonlinear plasma wave processes in the high- $\beta$ environment around the bow shock and the planetopause; and (c) the Interaction of Phobos dust with the solar wind. The existences of the dust torus along the Phobos orbit and its relevant plasma torus have been theoretically discussed. Krymskii et al. (1992) showed that local enhancement of electron density is to be expected in regions of the torus where the dust grains are positively charged. The wave observations can observe the dust and density enhancement.

Generation and propagation of electromagnetic waves: (a) Electromagnetic waves from the foreshock and the planetopause such as the $2 f_{p}$ emission and continuum radiation; and (b) Electromagnetic waves from the ionosphere and atmosphere. Recent observations by Mars Global Surveyor have shown the existence of strong magnetic field up to $400 \mathrm{nT}$ which is thought to be of crustal origin (Showstack, 1997). Such a magnetic field would make the lower ionospheric plasma a magneto-ionic medium, and, therefore, whistler-mode waves in the LFA band could be expected to propagate through the ionosphere. Impulsive emissions from the dust storm in the atmosphere and lightning discharges are both possible sources of whistlers. Planetary lightning has been proposed on Venus (Strangeway, 1991) based on the observations of Venusian impulsive low frequency waves.

Plasma waves above the ionosphere: Little is known observationally about wave-particle interactions in the region just above the Martian ionosphere. Theoretical studies have shown that the dominant physical process above the ionosphere is the interaction of planetary cold ions with shocked solar wind which is likely to lead plasma wave instabilities (Szegö et al., 1986, and references therein). As for the intense wave activity in a broad frequency observed in the planetosheath (or magnetosheath), Sagdeev et al. (1990) suggested that the cold, heavy Martian ions penetrate into the magnetosheath and form a cold beam where the dominant plasma is the shocked solar wind. Sagdeev et al. also suggested the possibility of a whistler instability to explain the Phobos 2 observations (Grard et al., 1989) of discrete bursts of plasma waves in the planetosphere. These waves can be interpreted in terms of a whistler-mode instability driven by the high energy electron tail. It is of benefit to compare the plasma waves in the Martian planetosheath with the ones in the Venusian mantle because it is dominated with the planetary $\mathrm{O}^{+}$ions appearing as a beam relative to the flowing ionosheath plasma. Huba (1993) suggested that the waves observed by the PVO spacecraft in the ionosheath (Scarf et al., 1980) are due to the Doppler shifted ion acoustic instability driven by the ion beam.

Plasma parameters and wave activity in the nightside ionosphere and tail: (a) Plasma structures and convection electric fields in the nightside region; and (b) Plasma waves and their contribution to particle acceleration and heating in

\section{Antenna \\ Main Electronics}



Fig. 1. System block diagram of the Low Frequency plasma wave Analyzer (LFA) onboard the PLANET-B spacecraft. 
the auroral-like region. Phobos 2 spacecraft observed a tail and neutral sheet structure (Nairn et al., 1991). The electrons may originate from the Martian ionosphere and be convected into the night sector. The variations in the electron density are possibly controlled by changes in the solar wind dynamic pressure. Enhanced wave activities over a wide range of frequencies up to several kilohertz were observed coincident with the high-density cold electrons. The possibility of a trough structure related with the draped solar wind magnetic field and acceleration to produce the energetic, heavy ion beams in the tail (Grebowsky et al., 1981; Taylor et al., 1985; Lundin et al., 1990), should be

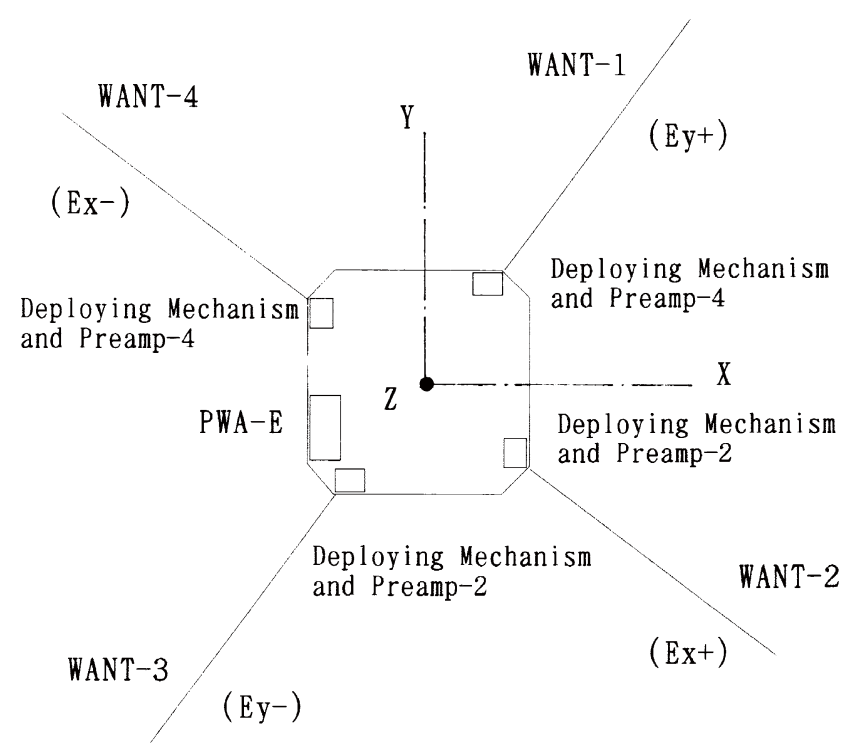

(Top View)

Fig. 2. Configuration of wire dipole antennas (WANT) deployed on the PLANET-B spacecraft. investigated.

Comparison with other planets: In order to compare the response of Martian exosphere to solar wind ram pressure with other planets, it is important to measure the position and structure of the bow shock, as used as the characteristics of the up- and down-stream regions. To this end, the global mapping of wave activity in these regions is necessary. The Phobos 2 spacecraft observed several events with enhanced plasma waves in the foreshock region. These waves are thought to be exited by a beam instability associated with suprathermal electrons and ions that are reflected and accelerated at the bow shock and stream back from it (Trotignon et al., 1991). Such a situation resembles the cases in the terrestrial and Venusian foreshock regions. Detailed maps of foreshock waves are given by Crawford et al. (1993) for Venus, and by Greenstadt et al. (1995) and Kasaba et al. (1997) for Earth.

\section{Instrument Characteristics}

The LFA measures the electric field received by the electric wire antennas (WANT) in three frequency bands: (1) $\mathrm{H}(10 \mathrm{~Hz} \sim 32 \mathrm{kHz}),(2) \mathrm{M}(10 \mathrm{~Hz} \sim 1 \mathrm{kHz})$, and (3) L (dc $\sim 40 \mathrm{~Hz}$ ). These signals are independently converted to spectra and waveforms by the onboard frequency analyzer (OFA) and the waveform capture (WFC) under operation of a central processing unit (CPU) and a digital signal processor (DSP). The LFA also measures the spacecraft potential with respect to the ambient plasma using the single probe (SPB) function. The block diagram of LFA is shown in Fig. 1. In order to concentrate on the description of the LFA, the components of PWS system and the power supply system are not shown.

\subsection{Electric antenna and preamplifier}

The LFA uses two wire dipole antennas that are orthogonally extended in a plane perpendicular to the spacecraft spin axis as shown in Fig. 2. Each dipole antenna (50 $\mathrm{m}$ tip-to-tip) consists of two wire elements, each $25 \mathrm{~m}$ long.

Want element

Pre amplifier

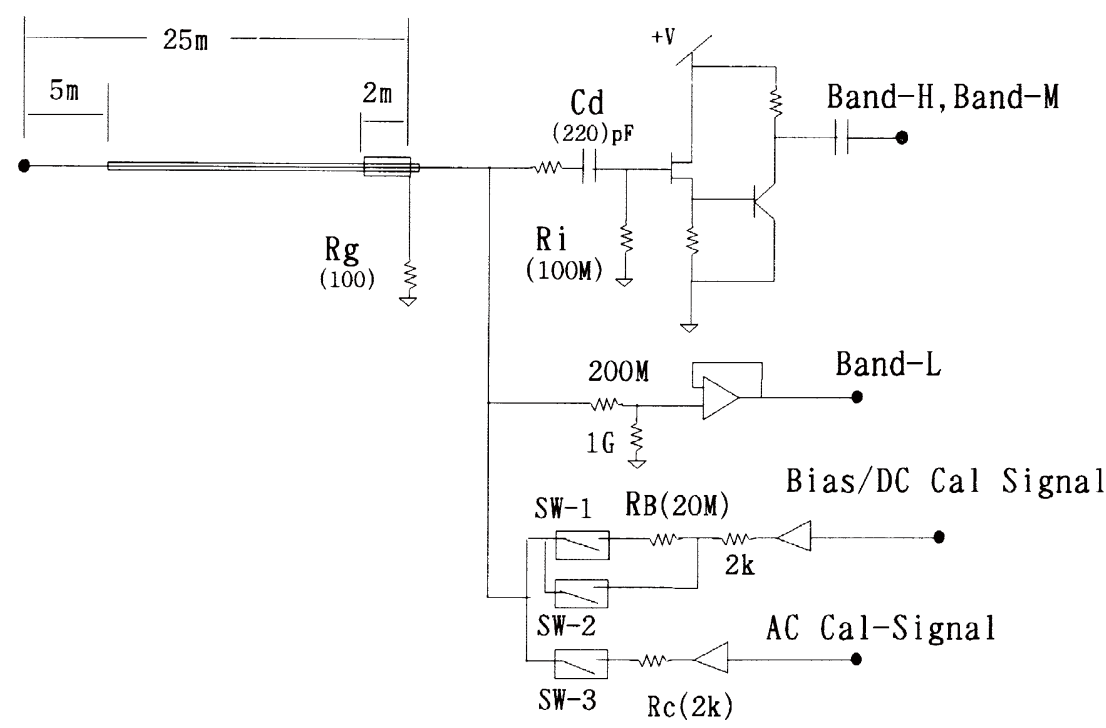

Fig. 3. Configuration of a wire antenna element with the coupling circuit of the LFA preamplifier. 
The wire elements are coated with a thin Polyimid film for electrical insulation from the ambient plasma except for the last $5 \mathrm{~m}$ at the tip of the elements. The first $2 \mathrm{~m}$ of each element (i.e., the $2 \mathrm{~m}$ closest to the antenna deploying mechanism) are shielded by a copper-mesh sleeve which is connected to the 2 nd-common of the preamplifier through a resistor $R_{g}(100 \Omega)$ to suppress the electromagnetic interference from the electrical materials on the spacecraft. Such a conductive sleeve is also effective in reducing the charging effect of insulation part of the Polyimid film. The preamplifiers are placed close to the antenna deploying mechanisms that are mounted in the side panels of the spacecraft. Figure 3 shows the configuration of a wire element, the circuit of the preamplifiers for the WANT-1 and WANT-3, and calibration and bias-current circuits. This type of wire antenna configuration was used on the GEOTAIL spacecraft for the measurements of terrestrial plasma waves (Matsumoto et al., 1994).

The electric potential difference between the WANT element and the spacecraft is fed to an FET source-follower with input resistor $R_{i}(100 \mathrm{M} \Omega)$ through a decoupling capacitor $C_{d}(220 \mathrm{pF})$ and sent to the $\mathrm{H}$ and $\mathrm{L}$ band receivers. The low frequency cutoff of the source-follower is $10 \mathrm{~Hz}$. The output of the source-follower is shared with the PWS system. The signal at frequencies from dc to $40 \mathrm{~Hz}$ is measured by an operational amplifier. It is connected to the wire antenna through a voltage-divider circuit $(200 \mathrm{M} \Omega$ and $1 \mathrm{G} \Omega$ ) and its output signal is fed to the $\mathrm{L}$ band receiver. A bias current that is necessary for the measurement of spacecraft potential is fed through a resistor $R_{B}(20 \mathrm{M} \Omega)$ to both the WANT-1 and -3 elements by turning on the switch (SW-1). The dc-calibration signal is fed by turning on the switch (SW-2). Neither the dc-calibration signal nor the bias current is fed to WANT-2 and WANT-4. In order to calibrate the overall gain and phase characteristics of all the subsystems, an ac calibration signal is fed through a resistor $R_{c}$ $(2 \mathrm{k} \Omega)$. The ac calibration signal is a composite signal of four square waves of fundamental frequencies of $10 \mathrm{~Hz}, 100$ $\mathrm{Hz}, 1 \mathrm{kHz}$ and $10 \mathrm{kHz}$. It is fed to all WANT antennas by turning on the switch (SW-3).

\subsection{Main electronics package}

The OFA calculates the electric field voltage spectrum from one of the dipole antennas (WANT-Ex or WANT-Ey) from $10 \mathrm{~Hz}$ to $32 \mathrm{kHz}$ (band-H and $-\mathrm{M}$ ). The signal from the antenna is sampled by the OFA-A/D converter with a sampling frequency of $100 \mathrm{kHz}$, coded into 16-bit string data and then analyzed by a digital signal processor (DSP: SMJ320C31) using the Fast Fourier Transformation (FFT) of 1024 points. The OFA has two output formats, the frequency-mode and time-mode. The frequency-mode provides the data with high frequency resolution averaged in time, while the time-mode provides the data with high time resolution averaged in frequency. The frequency steps of both modes are pseudo-logarithmically spaced. Data from both modes can be simultaneously transmitted and the spectral components are arranged in a variety of packet forms depending on the telemetry rate and the wave phenomena of interest.

The time-mode of the OFA-H can be replaced with the solar wind mode. In this mode, the spectral components are scanned and the narrowband peak in $1-32 \mathrm{kHz}$ is detected in order to monitor the plasma frequency and strength of the Langmuir wave in the space around Mars at fine time resolution. Several spectral components around the frequency of peak intensity are transmitted to confirm the real spectral peaking. This mode is effectively used when the data transmission rate is below $8 \mathrm{~Kb} / \mathrm{s}$. If the DSP does not work and the LFA can not provide the OFA data, the LFA can operate in an emergency mode in which the OFA data are replaced with the WFC-M data.

The WFC consists of the WFC-H, WFC-M, and WFC-L to provide waveforms from dc to $32 \mathrm{kHz}$. These waveforms are used for a detailed analysis of the plasma wave characteristics such as the wave normal direction and polarization. It is important to evaluate the wave characteristics in the plasma frame rather than those in the rest frame of spacecraft. Therefore, simultaneous measurements of LFA and the plasma apparatus are needed.

The WFC-H captures the waveform in the band- $\mathrm{H}(10 \mathrm{~Hz}$ to $32 \mathrm{kHz}$ ) from the WANT-Ex or WANT-Ey antenna. The duration of a waveform capture is $10.24 \mathrm{msec}$ and the sampling frequency is $100 \mathrm{kHz}$, coded into 16-bit string data. The data are stored in the WFC-H memory which is a 256 KByte block in the 4 MByte WFC-M memory, so that 128 samples can be recorded. The DSP also uses the waveform data for the FFT analysis so that the spectrum is provided by the OFA-H simultaneously.

The WFC-M captures simultaneously the waveforms in the $\mathrm{M}$ band $(10 \mathrm{~Hz}$ to $1 \mathrm{kHz})$ from both the WANT-Ex and WANT-Ey antennas. The waveforms are sampled $2.56 \mathrm{kHz}$ for each channel, coded into 14-bit string data, which can be compressed into 8-bit string data by the $\mu$-CODEC algorithm. The duration of waveform recording in the WFC-M

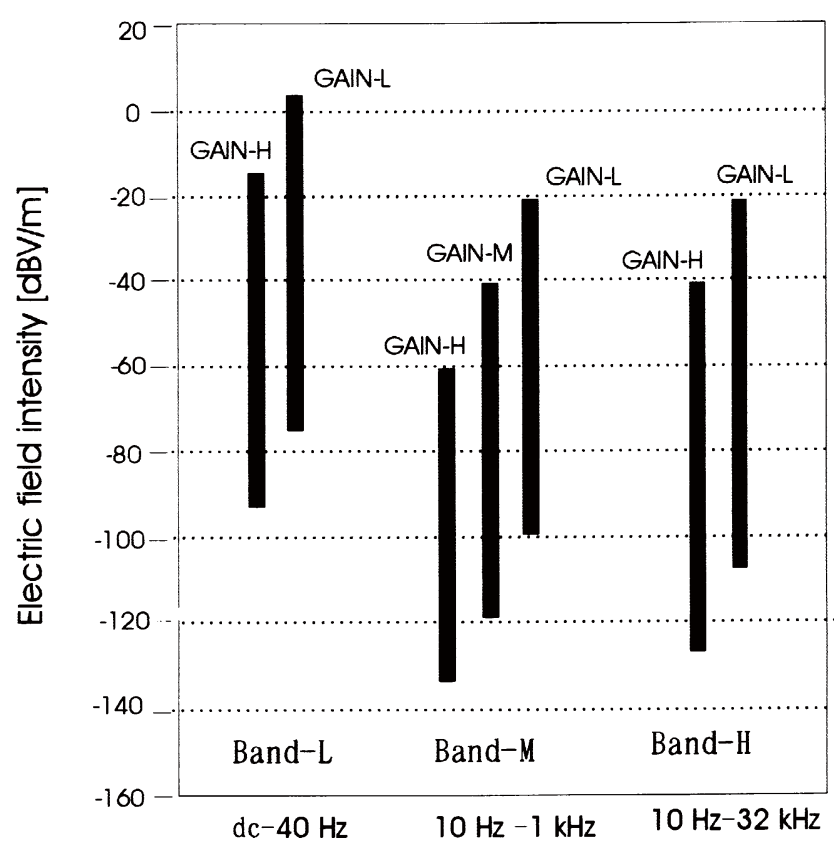

Fig. 4. Dynamic range of the $\mathrm{M}$, and $\mathrm{L}$ band receivers. The vertical bars indicate the intensity range of the electric field to be received. In deriving the intensity, the antenna effective length of $23 \mathrm{~m}$ and a coupling loss of $12 \mathrm{~dB}$ are used. The unit of electric field intensity is $(\mathrm{dBV} / \mathrm{m})$ as shown at the left side of the figure. 
Table 1. Function and the major specifications of LFA.

\begin{tabular}{|c|c|c|c|c|}
\hline Band & Function & Antenna & $\begin{array}{l}\text { Dynamic range } \\
\text { (gain stepped up) }\end{array}$ & Sampling frequency and resolution \\
\hline $\begin{array}{l}\text { Band-H } \\
10 \mathrm{~Hz} \sim 32 \mathrm{kHz}\end{array}$ & Spectrum and waveform & Ex or Ey & $70 \mathrm{~dB}(20 \mathrm{~dB})$ & $100 \mathrm{kHz}, 16$-bit \\
\hline $\begin{array}{l}\text { Band-M } \\
10 \mathrm{~Hz} \sim 1 \mathrm{kHz}\end{array}$ & Spectrum and waveform & Ex and Ey & $70 \mathrm{~dB}(20$ or $40 \mathrm{~dB})$ & $2.56 \mathrm{kHz}, 14-\mathrm{bit}$ \\
\hline $\begin{array}{l}\text { Band-L } \\
\text { dc } \sim 40 \mathrm{~Hz}\end{array}$ & Waveform and spacecraft potential & Ey & $70 \mathrm{~dB}(20 \mathrm{~dB})$ & $128 \mathrm{~Hz}, 14-$ bit \\
\hline
\end{tabular}

memory is 13.5 minutes. The DSP also uses the waveform data for the FFT analysis, so that the spectrum is provided by the OFA-M simultaneously. The data recorded in the WFC$\mathrm{H}$ and WFC-M memory can be transferred at a rate of 80 Byte/s in case of $2 \mathrm{~Kb} / \mathrm{s}$ down-link telemetry speed to Earth, i.e., for 54 minutes at the whole WHC-H memory and for 14.2 hours at the whole WFC-M memory. At the ground operation center, we can see the OFA-M spectrum in real time by using the Quick-Look System and we can determine whether the waveform should be dumped or not. Then we can select the address of memory to be reproduced by commands, judging from the spectra obtained by the OFA$M$ spectral data. Such a preview of spectrum of the captured waveform on memory will be of great benefit to reduce the time of dump mode operation and increase the usefulness of the waveform measurements.

The WFC-L can be used in a double probe mode or a single probe mode. In the double probe mode (DPB), the WFC-L measures the waveform from the WANT-Ey antenna over the frequency range from dc to $40 \mathrm{~Hz}$. The signal from the antenna is sampled with a sampling frequency of $128 \mathrm{~Hz}$ and coded into 14-bit string data, which can be compressed by the $\mu$-CODEC algorithm into 8-bit string data. In the single probe mode (SPB), the WFC-L measures the potential difference in the range from $-10 \mathrm{~V}$ to $+10 \mathrm{~V}$ between the spacecraft and the WANT-1 antenna element. A proper amount of bias current is fed by command to the antenna so that the antenna potential is close to that of the ambient plasma. Thus, the WFC-L provides the spacecraft potential relative to the ambient plasma, which can be used to estimate the plasma number density in the Martian dilute plasma. Because of the attitude control of the spacecraft, the sunlit area of the WANT elements is kept constant at any spacecraft spin phase, and no shadow of spacecraft is formed on the WANT elements. The wire antenna is longer than the Debye length of the plasma on the most of the PLANET-B elliptical orbit. This antenna configuration is good for precise measurement of potential difference. At the low bit rate modes, the data transmission rate of the WFC-L becomes less than the sampling rate of $128 \mathrm{~Hz}$ in both modes. In these cases, the digital low-pass filter is operated by the DSP with cut-off frequencies of $1 \sim 32 \mathrm{~Hz}$ in order to avoid the aliasing.

The $\mathrm{H}$ and $\mathrm{L}$ band receivers have a dynamic range of $\sim 70$ $\mathrm{dB}$ and their gain can be stepped up by 0 or $20 \mathrm{~dB}$. Therefore, the effective dynamic range reaches $\sim 90 \mathrm{~dB}$. The $\mathrm{M}$ band receiver has a dynamic range of $\sim 70 \mathrm{~dB}$ and the gain can be stepped up by 0,20 , or $40 \mathrm{~dB}$. Therefore, the effective dynamic range reaches $\sim 110 \mathrm{~dB}$. We anticipate that the wave amplitudes can dramatically change as the spacecraft orbits Mars, but the real-time operations from the Earth are impossible. In order to avoid saturation and insufficient gains, therefore, the three receivers have an automatic gain control (AGC) operated by the CPU. The conditions for the stepping up and down of the gain depend on wave amplitudes at specific time intervals observed in each frequency band. These conditions are selected by commands. Table 1 summarizes the major specifications of LFA.

Figure 4 show the estimated intensity $(\mathrm{dBV} / \mathrm{m})$ of the electric field measured by each receiver. As seen in Fig. 4, the dynamic range of LFA covers the wave intensity observed by the Phobos 2 spacecraft (Trotignon et al., 1991), and it is almost comparable to the wave receivers used in the Earth's magnetospheric observations (Gurnett, 1995). In order to make this estimate, the effective length of the dipole antenna,


except the shielded $2 \mathrm{~m}$-part. The coupling loss in the stage of the antenna and the preamplifier is determined by the antenna impedance, $Z_{\text {ant }}$, in the space plasma (which is assumed to be $200 \mathrm{pF}$ ) and the input impedance $Z_{\mathrm{i}}$ (about 560 $\mathrm{pF}$ and $200 \mathrm{M} \Omega$ in parallel) of the preamplifier including the capacitance of the shielding.

\section{Concluding Remarks}

The Low Frequency plasma wave Analyzer, LFA, on board the PLANET-B spacecraft has been designed to measure Martian plasma waves in the band from dc to 32 $\mathrm{kHz}$ and the spacecraft potential relative to the ambient plasma by using the long wire dipole antennas with improved signal-to-noise ratio. By combining the DSP and CPU, signal-processing functions such as FFT, anti-aliasing filtering, AGC and others are greatly enhanced, while the weight, volume, and power consumption are reduced. With the capability of performing both spectral measurements and waveform capture using a 4 MByte memory, the LFA will address many of the scientific objectives of the PLANETB spacecraft. LFA data can be used to determine plasma parameters from the solar wind to the ionosphere and their boundaries, to examine the role of plasma waves in the mass-loading process, the microscopic plasma phenomena induced by the Martian interaction with the solar wind, waves related with the moon Phobos, the generation and propagation of electromagnetic waves from the atmosphere through the ionosphere, the plasma structure and wave modes in the nightside ionosphere and tail, and finally, can 
be used to compare Martian plasma waves with those of other planets such as non-magnetized Venus and magnetized Earth.

Acknowledgments. The LFA team members express their sincere thanks to Prof. T. Yamamoto of the Institute of Space and Astronautical Science and all PLANET-B mission members. The PWA/LFA hardware was manufactured by Meisei Electric Company. We would like to note contributions by Mr. T. Toda of Matsushita Co. Ltd. and Mr. K. Shin of Kyoto Univ., RASC.

\section{References}

Crawford, G. K. et al., VLF imaging of the Venus foreshock, Geophys. Res. Lett., 20, 2801-2804, 1993.

Dubinin, E. et al., Comparison of observed plasma and magnetic field structures in the wakes of Mars and Venus, J. Geophys. Res., 96, 1118911197, 1991.

Grard, R. et al., First measurements of plasma waves near Mars, Nature, 341, 607-609, 1989.

Grard, R. et al., Plasma and waves around Mars, Planet. Space Sci., 39, 89-98, 1991.

Grebowsky, J. M. et al., Venus nighttime ionospheric holes: the signatures of parallel electric field acceleration regions?, Geophys. Res. Lett., 8 1273-1276, 1981.

Greenstadt, E. W. et al., Spatial distribution of electron plasma oscillations in the Earth's foreshock at ISEE 3, J. Geophys. Res., 100, 1993319939, 1995

Gurnett, D. A., The polar plasma wave instrument, Space Sci. Rev., 71, 597$622,1995$.

Huba, J. D., Generation of waves in the Venus mantle by the ion acoustic beam instability, Geophys. Res. Lett., 20, 1751-1754, 1993.

Johnson, F. S. et al., Viking 1 electron observations at Mars, J. Geophys. Res., 96, 11097-11118, 1991.

Kasaba et al., GEOTAIL observation of $2 f_{p}$ emission around the terrestrial electron foreshock, Adv. Space Res., 20, 699-702, 1997.

Krymskii, A. M. et al., The electromagnetic effects of the solar wind interaction with the Phobos neutral gas halo and dust torus, Planet. Space Sci., 40, 1033-1041, 1992.

Lundin, R. et al., ASPERA/PHOBOS measurements of the ion outflow from the Martian ionosphere, Geophys. Res. Lett., 17, 873-876, 1990. Matsumoto, H. et al., Plasma wave observations with GEOTAIL spacecraft, J. Geomag. Geoelectr., 46, 59-95, 1994.

Moses, S. L. et al., Characteristics of spectra from the Martian bow shock and comparison with Venus, Earth, AMPTE, Jupiter, and Saturn, $J$. Geophys. Res., 96, 11221-11226, 1991.

Nairn, C. M. C. et al., Plasma wave observations in the night sector of Mars, J. Geophys. Res., 96, 11227-11233, 1991.

Pedersen, A. et al., Deviation of electron densities from differential potential measurements upstream and down stream of the bow shock and in the magnetosphere of Mars, J. Geophys. Res., 96, 11243-11252, 1991.

Sagdeev, R. Z. et al., Wave activity in the neighborhood of the bow shock of Mars, Geophys. Res. Lett., 17, 893-896, 1990.

Scarf, F. L. et al., Pioneer Venus plasma wave observations: The solar wind-Venus interaction, J. Geophys. Res., 85, 7599-7612, 1980.

Showstack, R., Mars has crustal, complex magnetic field, EOS, 78, 40, 429, 1997.

Slavin, J. A. et al., The solar wind interaction with Mars: Mariner 4, Mars2, Mars3, Mars5, and Phobos 2 observations of bow shock position and shape, J. Geophys. Res., 96, 11235-11241, 1991.

Strangeway, R. J., Plasma waves at Venus, Space Sci. Rev., 55, 275-316, 1991.

Szegö, K. et al., Electron acceleration above the dayside ionopause of non magnetized bodies, Pros. 4th COSPER Colloquium, edited by T. I. Gombosi, pp. 247-252, Pergamon Press, 1986.

Taylor, H. A. et al., Venus nightside ionospheric troughs: Implications for evidence of lightning and volcanism, J. Geophys. Res., 90, 74157426, 1985.

Trotignon, G. et al., Plasma wave system measurements of the Martian bow shock from the Phobos 2 spacecraft, J. Geophys. Res., 96, 11253$11264,1991$.

Woo, R. and A. J. Kilore, Magnetization of the ionospheres of Venus and Mars: results from radio occultation measurements, J. Geophys. Res., 96, 11073-11081, 1991.

H. Matsumoto, T. Okada (e-mail: okada@pu-toyama.ac.jp), K. Hashimoto, I. Nagano, S. Yagitani, M. Tsutsui, Y. Kasaba, K. Tsuruda, H. Hayakawa, A. Matsuoka, S. Watanabe, H. Ueda, I. Kimura, Y. Kasahara, Y. Omura, T. Matsumura, T. Imachi, K. Ishisaka, and Y. Tateno 